\title{
Topology evolution during coarsening of nanoscale metal network structures
}

\author{
Yong Li, ${ }^{1,2}$ Bao-Nam Dinh Ngô, ${ }^{2}$ Jürgen Markmann, ${ }^{2,1}$ and Jörg Weissmüller ${ }^{1,2, *}$ \\ ${ }^{1}$ Institute of Materials Physics and Technology, Hamburg University of Technology, Germany \\ ${ }^{2}$ Institute of Materials Research, Materials Mechanics, Helmholtz-Zentrum Geesthacht, Germany
}

(Received 19 February 2019; published 31 July 2019)

\begin{abstract}
Many experiments exploit curvature-driven, surface-diffusion-mediated coarsening for tuning the characteristic structure size of metal network structures made by dealloying, such as nanoporous gold. Here we study this process by kinetic Monte Carlo simulation. The initial microstructures are leveled Gaussian random fields, approximating spinodally decomposed mixtures, of different solid fraction $\varphi$. Earlier work establishes these structures as valid representations of the nanoporous gold microstructure. We find that the coarsening law for the characteristic spacing between the ligaments of the network is universal, whereas the time evolution of the characteristic ligament diameter is not. The expected time exponent $1 / 4$ is confirmed by our simulation. Contrary to what may be expected based on continuum models, the degree of surface faceting or roughness has no apparent effect on the coarsening kinetics. In the time interval of our study, the network connectivity-as measured by a scaled density of topological genus-remains sensibly invariant for networks with $\varphi \geqslant 0.3$, consistent with previous reports of a self-similar evolution of the microstructure during coarsening. Yet, networks with lesser $\varphi$ lose their connectivity on coarsening and can even undergo a percolation-to-cluster transition. This process is slow for $\varphi$ only little below 0.3 and it accelerates in networks with lesser $\varphi$. The dependency of the connectivity evolution on $\varphi$ may explain controversial findings on the microstructure evolution of nanoporous gold in experimental studies.
\end{abstract}

DOI: 10.1103/PhysRevMaterials.3.076001

\section{INTRODUCTION}

Nanoscale metal network materials made by dealloying, and notably nanoporous gold (NPG), are under investigation as model systems for fundamental studies of small-scale plasticity [1]. These studies have provided insights into the impact of size [2-7] and of surface effects [8-10] on small-scale plasticity and they highlight the role of nanoporosity in stress corrosion cracking [11,12]. The discussion tends to focus on the ligament size, $L$, and on the solid (volume) fraction, $\varphi$, as the defining microstructural parameters. Yet, inconsistencies between published data for strength or stiffness suggest that additional parameters might be relevant for the mechanical behavior [1,13-15]. It is therefore significant that the connectivity - to be defined below - of the network has been highlighted as an additional, important characteristic [16] which strongly affects the mechanical behavior [14,17-19].

With attention to as-dealloyed nanoporous metal, it has indeed been demonstrated that the anomalously low effective Young's modulus $[14,20]$ can be understood as the consequence of a decrease in connectivity with decreasing $\varphi$ [21]. A standard processing approach in experimental studies is to establish series of samples with ever-larger $L$ by coars-

\footnotetext{
*Corresponding author: weissmueller@tuhh.de

Published by the American Physical Society under the terms of the Creative Commons Attribution 4.0 International license. Further distribution of this work must maintain attribution to the author(s) and the published article's title, journal citation, and DOI.
}

ening [22]. The underlying mechanism is curvature-driven redistribution of matter by surface diffusion $[16,23,24]$, with the pinch-off of ligaments by Plateau-Rayleigh instability as the topology-changing events [16]. Model microstructures for atomistic and finite-element model studies of NPG have been generated by simulating spinodal decomposition $[17,20,25,26]$, which can also involve coarsening. Since the connectivity affects the mechanics, one is led to ask how will the connectivity evolve during coarsening?

The experimental findings on the above issue are contradictory. Changes in Young's modulus during coarsening suggest that the fraction of disconnected ligaments increases strongly as the ligaments grow [14]. Yet the analysis of experimental tomographic reconstructions of NPG appears to refute the suggestion: These data suggest that the coarsening is self-similar; in other words, the number of connections in a representative volume element is a constant, independent of the ligament size [15,27].

In view of the similarity between the microstructures of NPG and of spinodally decomposed mixtures [20,21,25,26], phase-field simulation studies of coarsening during spinodal decomposition may provide a lead to a possible origin of the contradictory findings. Such studies find that symmetric spinodal structures, with equal phase fractions, retain their connectivity during coarsening [28]. Yet they also reveal that the evolution of the topology in asymmetric $(\varphi \neq 0.5)$ structures depends sensitively on $\varphi[29,30]$. Specifically, the spinodal decomposition of mixtures by bulk diffusion yields bicontinuous (percolating) and essentially self-similar microstructures when $0.36 \leqslant \varphi \leqslant \frac{1}{2}$; lesser values of $\varphi$ lead to disconnected clusters [29]. Apparently contradictory observations might 
then be obtained in studies of topology evolution that work with samples of different $\varphi$. Yet, even though similarities between NPG and spinodal microstructures are widely acknowledged, it is not obvious in how far the observations in Ref. [29] can be transferred to NPG. In stark contrast to those observations, the experiments on NPG show load-bearing and, hence, well-connected structures at $\varphi$ ranging from 0.45 down to as small as 0.25 [1]. This is not surprising in view of the quite different underlying processes. Spinodal decomposition involves, in its early stage, the continuous conversion of a uniform solution into a two-phase microstructure when composition fluctuations grow in amplitude. During dealloying, by contrast, the two-phase microstructure is created by the progression of a discontinuous interface between the solid and the corrosive medium $[31,32]$. In each case the geometric arrangement of the two phases is correlated, not random, and this affects the criteria for percolation. The detailed nature of such correlations depends on the nature of the active processes [33]. These processes are distinctly different between spinodal decomposition and dealloying. The use of phase-field generated spinodal structures as models for NPG may therefore require caution.

The evolution of the topology during coarsening of nanoporous nanoparticles by surface diffusion has been studied by kinetic Monte Carlo (KMC) simulation [16]. These particles evolve into massive solids with no connected porosity. In experiments, an analogous process results in denser layers near the surface of nanoporous metal [34]. The observations on the coarsening of nanoparticles cannot be transferred to bulk network structures, since the densification which is mediated by the surface of the particles has no equivalent in the interior of the network [35].

Simulations exploring the impact of the solid fraction on the evolution of network topology in the bulk, considering realistic network geometries and focusing on coarsening by surface diffusion, have not been reported so far. Here we present such a study, based on KMC simulations with parameters matched to NPG. We study realistic initial structures with solid fractions in the range covered by experiment, focusing on the behavior in the network's bulk as opposed to its external surface, and allowing for surface diffusion as the dominant transport mechanism.

\section{METHODS AND DEFINITIONS}

\section{A. Leveled-wave initial microstructures}

Our simulations ran on a rigid face-centered cubic (fcc) lattice with lattice parameter $a=408 \mathrm{pm}$. Unless otherwise stated, the simulation boxes extended over 200 lattice parameters in each direction. With four sites per fcc unit cell, this corresponds to 32 million sites and to a simulation box edge length $b=81.6 \mathrm{~nm}$. Starting configurations were generated as leveled Gaussian fields, using a model for the microstructure at the end of early-stage spinodal decomposition [36] in its adaptation for periodic boundary conditions, as described in detail in Ref. [21]. In brief, plane waves with wave vectors

$$
\mathbf{q}_{i}=\frac{2 \pi}{b}\left(h_{i} \mathbf{e}_{1}+k_{i} \mathbf{e}_{2}+l_{i} \mathbf{e}_{3}\right),
$$

of same magnitude, $q$, but different direction and with random phase shifts, were superimposed to map a random field onto the lattice. Here $\left\{\mathbf{e}_{i}\right\}$ provides an orthonormal basis in the reciprocal space and the tuples $\left(h_{i}, k_{i}, l_{i}\right)$ consist of integers and are of fixed magnitude, $H$. In other words, $H=$ $\sqrt{h_{i}^{2}+k_{i}^{2}+l_{i}^{2}}=$ const. The field was then binarized into solid and pore by a level cut selected for the desired solid volume fraction $\varphi$.

Random networks generated in this way provide a realistic representation of as-dealloyed NPG: Their Young's modulus is isotropic and in excellent agreement with experiments covering $\varphi=0.25-0.45$ [21]. Furthermore, their connectivity density agrees with experimental data obtained from tomographic reconstructions of NPG [15,21]. A pronounced interference peak in the experimental small-angle structure factor of NPG $[37,38]$ confirms the presence of a dominant characteristic wavelength, as in the leveled wave model.

\section{B. Coarsening by kinetic Monte Carlo simulation}

Coarsening was studied by on-lattice KMC simulations [39] with periodic boundary conditions in 3D, using the open source codes SPPARKS [40] for KMC and OVITO [41] for visualization. A nearest-neighbor bond counting model supplies the energy, $\mathfrak{E}$, of each lattice site, namely for a vacancy $\mathfrak{E}=0$ and for atoms $\mathfrak{E}=\varepsilon z$ with $z$ the nearest-neighbor coordination and $\varepsilon$ half of the bond energy. At each simulation step, an atom may jump to one of its neighboring vacancies. Each jump induces a change, $\Delta E$, in the total energy. This change relates to $\Delta z$, the difference between final and initial coordination number of the jumping atom, by $\Delta E=2 \varepsilon \Delta z$. The rate, $r$, of such an event is

$$
r=\left\{\begin{array}{ll}
v & \text { if } \Delta E<0 \\
v \exp \left[-\Delta E /\left(k_{B} T\right)\right] & \text { if } \Delta E \geqslant 0
\end{array},\right.
$$

where $v, k_{B}$, and $T$ are the attempt frequency, Boltzmann's constant, and the temperature, respectively. Transition state theory describes the dependency of $v$ on details of the atomicscale diffusion mechanism; at $T=900 \mathrm{~K}$ a value of $10^{12} \mathrm{~s}^{-1}$ is typically assumed [40,42].

At $T=900 \mathrm{~K}$, each of the simulations with the box edge length $200 a$ typically required $2000 \mathrm{~h}$ of CPU time on each of the 64 cores used in parallel, about $130000 \mathrm{~h}$ of CPU in total. Runs at $1800 \mathrm{~K}$ were faster, about 28000 total CPU hours. A run at $1800 \mathrm{~K}$ with a $400 a$ edge length box and 256 cores required 98000 total CPU hours.

An obvious energy scale in our problem is provided by the specific surface excess free energy, $\gamma$, which is the driving force for coarsening. Densely packed surfaces of metal crystals typically exhibit $\gamma$ in the order of $1 \mathrm{~J} / \mathrm{m}^{2}$. Based on the excess energy in the broken bonds per area of surface, we find that our values of $\varepsilon$, namely $-0.15 \mathrm{eV}$, and $a$ imply specific surface energies of $1.00,1.16$, and $1.23 \mathrm{~J} / \mathrm{m}^{2}$, respectively, for $\{111\},\{100\}$, and $\{110\}$ terraces. These values are about $10 \%$ less than predictions by density functional theory (DFT) for gold [43], yet their ratios agree with DFT. 


\section{Extracting ligament size and connectivity}

Coarsening results from the strife to reduce the product of $\gamma$ with $A$, the area of surface. Thus, the specific (per volume, $V_{S}$, of the solid) surface area, $S_{V}=A / V_{S}$, is a natural microstructural parameter in studies of coarsening at conserved volume [29,44]. This parameter is also routinely determined in experiments on NPG $[9,45,46]$. In our study, the surface reconstruction algorithm of Ref. [47] supplied $A$ and $V_{S}$ as the basis for $S_{V}$. The algorithm is based on a Delaunay tessellation of the atomic structure of the solid. Extracting the location of a surface from the tessellation involves the convolution with a spherical probe volume [47], the radius of which was set to $0.3 \mathrm{~nm}$ in our study.

Since we are interested in the growth law of the network, we here used $A$ and $V_{S}$ of the largest cluster. Other, isolated clusters were ignored.

As a more descriptive parametrization of $S_{V}$, a characteristic length scale is provided by the apparent ligament size (diameter) $L_{\mathrm{ap}}$,

$$
L_{\text {ap }}=4 / S_{V} .
$$

Equation (3) applies to long circular rods as a rough approximation of the microstructure of NPG. The results of Ref. [21] imply an exact relation between $S_{V}$ and a different measure for size, namely the characteristic spacing, $\tilde{L}$, between the centers of neighboring ligaments:

$$
\tilde{L}=\alpha \lambda=\frac{4}{S_{V}} \frac{\alpha}{\sqrt{3} \varphi} e^{-\left[\operatorname{erf}^{-1}(2 \varphi-1)\right]^{2}}
$$

with $\alpha=1.23$ and $\lambda=2 \pi / q$ the wavelength underlying the Gaussian field. Equation (4) is exact for the initial leveledwave microstructure; it remains valid during coarsening if the microstructure evolution is self-similar.

The topological genus, $G$, may be identified with the number of connections in a network microstructure [15,16,24,48]. More precisely, $G$ represents the maximum number of connections in the network that may be cut (disconnected) before the structure disconnects into two independent ones and falls apart. By means of example, the values of $G$ for a sphere, a donut, and a pretzel are 0,1 , and 3 , respectively.

For a material with a given microstructure, $G$ scales with the sample volume. In the context of coarsening it is desirable to work with a ligament connectivity measure which is independent of sample volume and of microstructural length scale. Here we use a scaled genus, $g$, defined by [21]

$$
g=G \frac{\tilde{L}^{3}}{V_{\text {total }}},
$$

where $V_{\text {total }}$ represents the total sample volume, solid plus pores. By its definition, $g$ represents the number of connections in a representative volume element of size $\tilde{L}^{3}$. If the microstructure evolves in a self-similar manner during coarsening, then $g$ remains a constant.

As a numerical approach to the topology, we evaluated the Betti numbers, $B_{0}$ and $B_{1}$, of the KMC-generated structures. The Betti numbers are topological invariants of the surface of a microstructure $[49,50]$. They are readily computed by the open-source code CHomP [51], which we used with its option for periodic boundary conditions activated. $B_{0}$ represents the number of connected components, while $B_{1}$ measures the number of handles of the structure [49]. $B_{0}=1$ indicates that the solid forms a single connected cluster, whereas larger values of $B_{0}$ imply the formation of isolated particles. The genus is obtained as $G=B_{1}$.

\section{RESULTS}

\section{A. Temperatures and initial configurations}

Simulations were run at two temperatures, $T=900 \mathrm{~K}$ and $1800 \mathrm{~K}$. Runs at $T<900 \mathrm{~K}$ require prohibitive CPU time because the higher ones among the energy barriers for transport cannot be efficiently overcome at low $T$. At $T>$ $1800 \mathrm{~K}$, the vacancy concentration and the fraction of single atoms in the (lattice-) gas are no longer negligible and so bulk transport may start to contribute. Figures 1(a) and 1(b) show Wulff shapes of isolated particles equilibrated in simulations at $900 \mathrm{~K}$ and $1800 \mathrm{~K}$. At the lower temperature, the particle appears almost perfectly faceted. By contrast, the higher temperature leads to a rougher surface, with a substantial amount of surface defects. Comparing the simulations at the two temperatures will therefore provide insights into the impact of thermal roughening on the microstructure evolution.

Our simulations of coarsening were based on initial configurations, generated by the leveled-wave algorithm, with different solid fractions. Figure 1(c) shows an exemplary initial configuration, here for $\varphi=0.35$. The microstructure geometry resembles that of experimental NPG. The surfaces in this initial state are rough, which is a consequence of projecting the leveled random field onto the crystal lattice. This configuration used $H=\sqrt{449}$. Figure $1(\mathrm{~d})$ shows the 120 wave vectors, emphasizing that the orientation space is rather uniformly covered. This is consistent with the apparent absence of microstructural texture in Fig. 1(c).

\section{B. Evolution of characteristic size}

Figures 1(e) and 1(f) show configurations at the end of the $900 \mathrm{~K}$ and $1800 \mathrm{~K}$ coarsening runs. By construction, $\varphi$ is invariant during the coarsening. By contrast, the characteristic structure size has increased about fourfold. At the same time, the number of ligaments decreases from initially about $10^{4}$ to about 300 in the final state (see discussion of genus below). The almost tenthousand pinch-off events which underlie this structural evolution form the basis of our analysis. The coarsened structures in Fig. 1 have developed a texture, with ligaments preferentially oriented parallel to the edges of the simulation box, which are $\langle 100\rangle$ crystallographic directions.

Figure 2 shows subsets $(50 \times 50 \times 50$ lattice spacings $)$ of the larger simulation box, following their evolution. The columns of the figure refer to the two temperatures. The rough surfaces of the initial state are seen in the top row, Fig. 2(a). The central row [Figs. 2(b) and 2(c)] shows snapshots early in the relaxation, before the onset of substantial coarsening. It is seen that relaxation immediately leads to faceting, even for the smallest structures. The bottom row [Figs. 2(d) and 2(e)] shows the final states. The prevailing features are $\{111\}$ and $\{100\}$ terraces, with similar numbers of atoms in both. Large facets are apparent at the lower temperature, whereas the surfaces are rougher at higher temperature. 
(a)

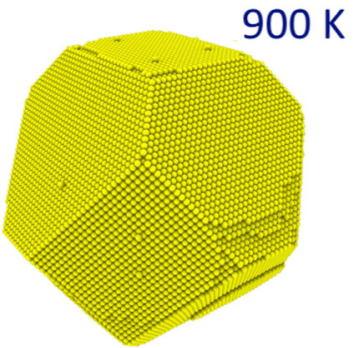

(c)
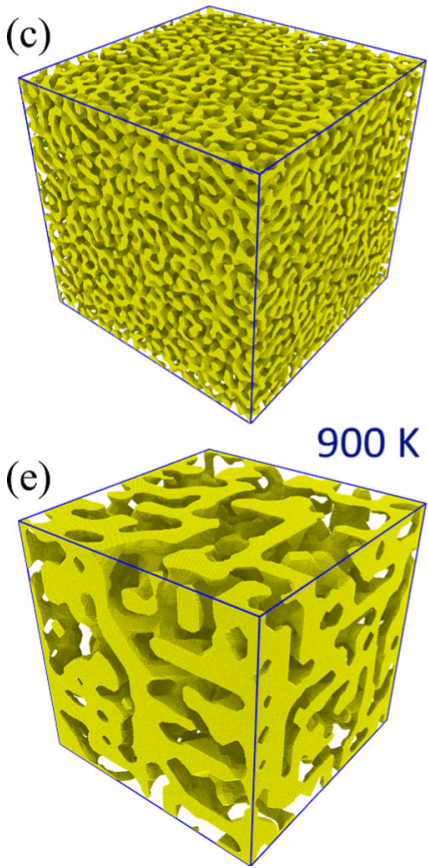

(b)

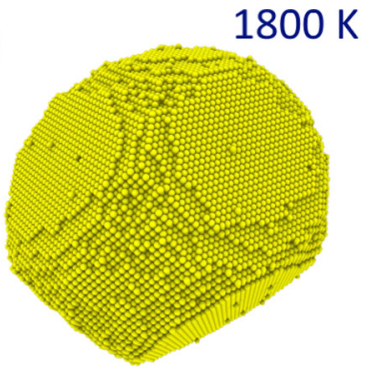

(d)
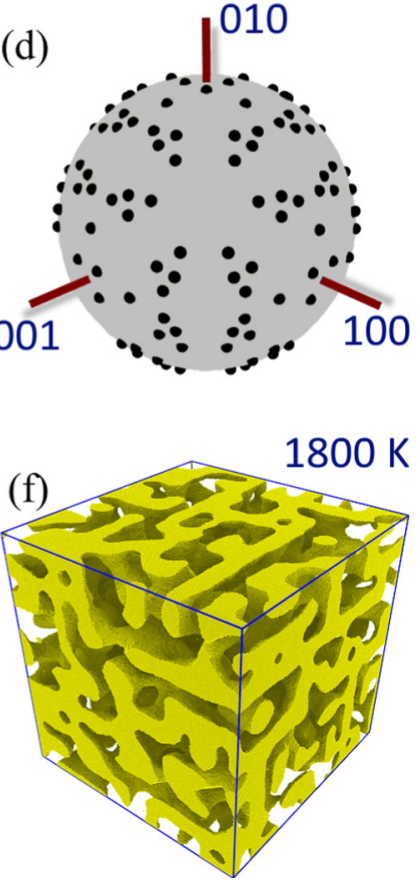

FIG. 1. Temperatures, initial and final configurations. The top row shows Wulff shapes of isolated particles equilibrated at temperatures $T=900 \mathrm{~K}$ (a) and $1800 \mathrm{~K}$ (b). Note the roughness at $1800 \mathrm{~K}$. Central row shows in (c) a simulation box with the initial, leveled-wave microstructure and in (d) the position of the underlying 120 wave-vector directions (black dots) on the unit sphere in reciprocal space. The 100-type crystallographic directions are indicated. The bottom row shows the coarsened structure at the end of the simulation runs at $900 \mathrm{~K}$ (e) and $1800 \mathrm{~K}$ (f). Note the appearance of microstructural texture after coarsening. Simulation box size in (c), (e), and (f) is $200 \times 200 \times 200$ face-centered cubic unit cells, phase fraction in the example is 0.35 . Yellow spheres denote atom positions.

We used different strategies for the initial configurations. The runs at $900 \mathrm{~K}$ compared systems with different $\varphi$ but identical apparent ligament diameter, $L_{\mathrm{ap}}$. This required selecting different values of $H$ and, therefore, different random fields with different characteristic $q$ and different characteristic spacing $\tilde{L}$. By contrast, the runs at $1800 \mathrm{~K}$ generated the structures with different $\varphi$ by level cuts on one and the same random field. Here the initial structures have the identical $\tilde{L}$ but different $L_{\text {ap }}$. For more details on the initial configurations see the Supplemental Material [52], confirming the validity of Eq. (4).

Figures 3(a) and 3(b) show how the size parameters $L_{\text {ap }}$ and $\tilde{L}$ evolve over time at $900 \mathrm{~K}$ and $1800 \mathrm{~K}$, respectively.
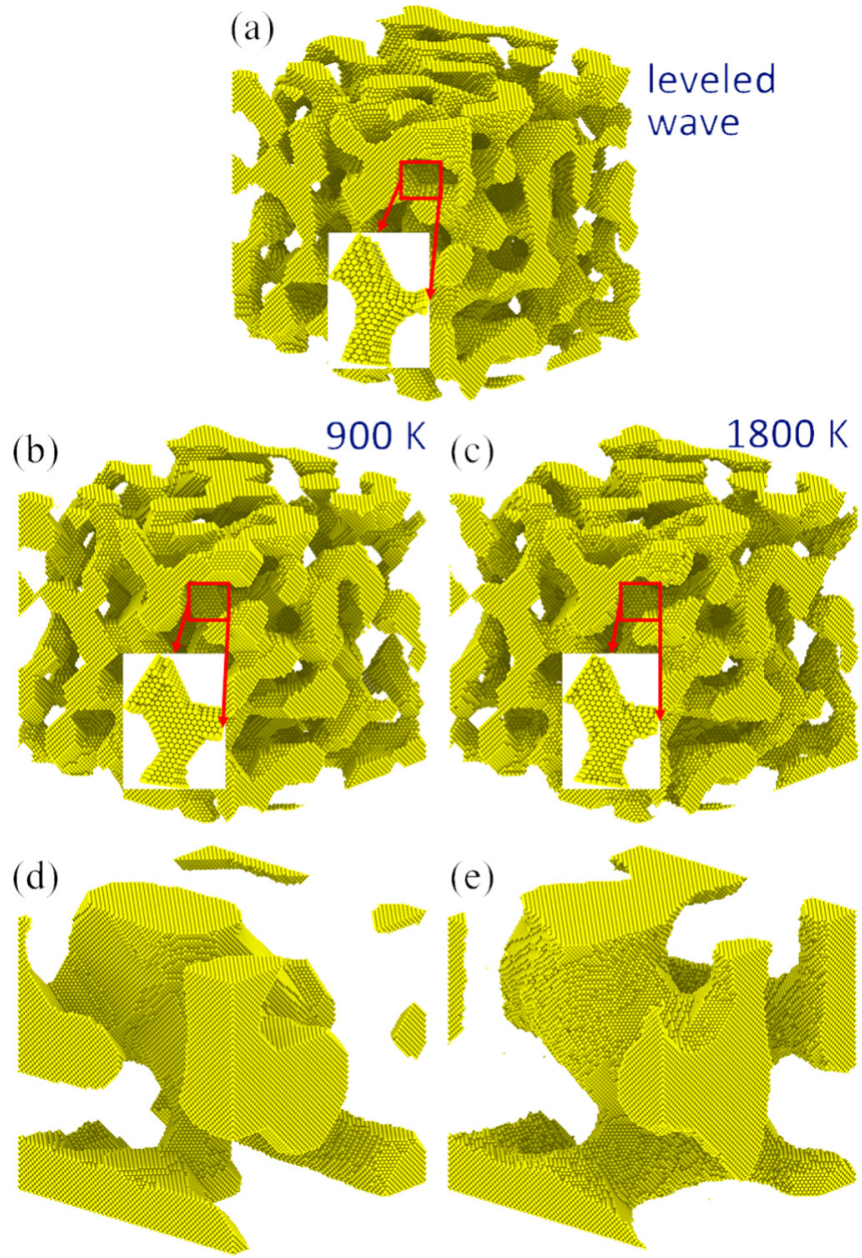

FIG. 2. Microstructure evolution during coarsening. (a) Initial structure generated by the leveled-wave construction. Central row: Structures after initial relaxation and before the onset of significant coarsening. (b) After period of $1.1 \times 10^{6} v^{-1}$ at temperature $T=900 \mathrm{~K}$; (c) after period of $10^{8} v^{-1}$ at $T=1800 \mathrm{~K}$. Insets in (a)-(c) show a blowup of an exemplary ligament, emphasizing initial roughness and faceting during relaxation. Bottom row: Coarsened structure at the end of the simulation runs at $T=900 \mathrm{~K}$ (d) and $1800 \mathrm{~K}(\mathrm{e})$. Note large facets at lower temperature and rougher surface at higher temperature. All subfigures show the same subset of the larger simulation box; subset size is $50 \times 50 \times 50$ lattice spacings. Solid fraction is 0.35 . Yellow spheres denote atom positions.

The size parameters were determined from the specific surface areas using Eqs. (3) and (4). Their initial values are systematically smaller, by $11 \pm 2 \%$, than the a priori known values (see the Supplemental Material [52]) of the leveled random fields. The deviation arises, first, because of the atomic-scale roughness of the initial surface-this increases $A$ over the smooth surface of the leveled wave model. Second, the surface reconstruction algorithm lets the surface interpolate the atom centers. By construction, these are displaced inward from the leveled wave surface. $V_{S}$ is then underestimated. Apart from the systematic deviation, the sizes provide an accurate match with the leveled-wave template.

Figures 3(a) and 3(b) reveal three stages of size evolution for each simulation run. In the first stage, at extremely 

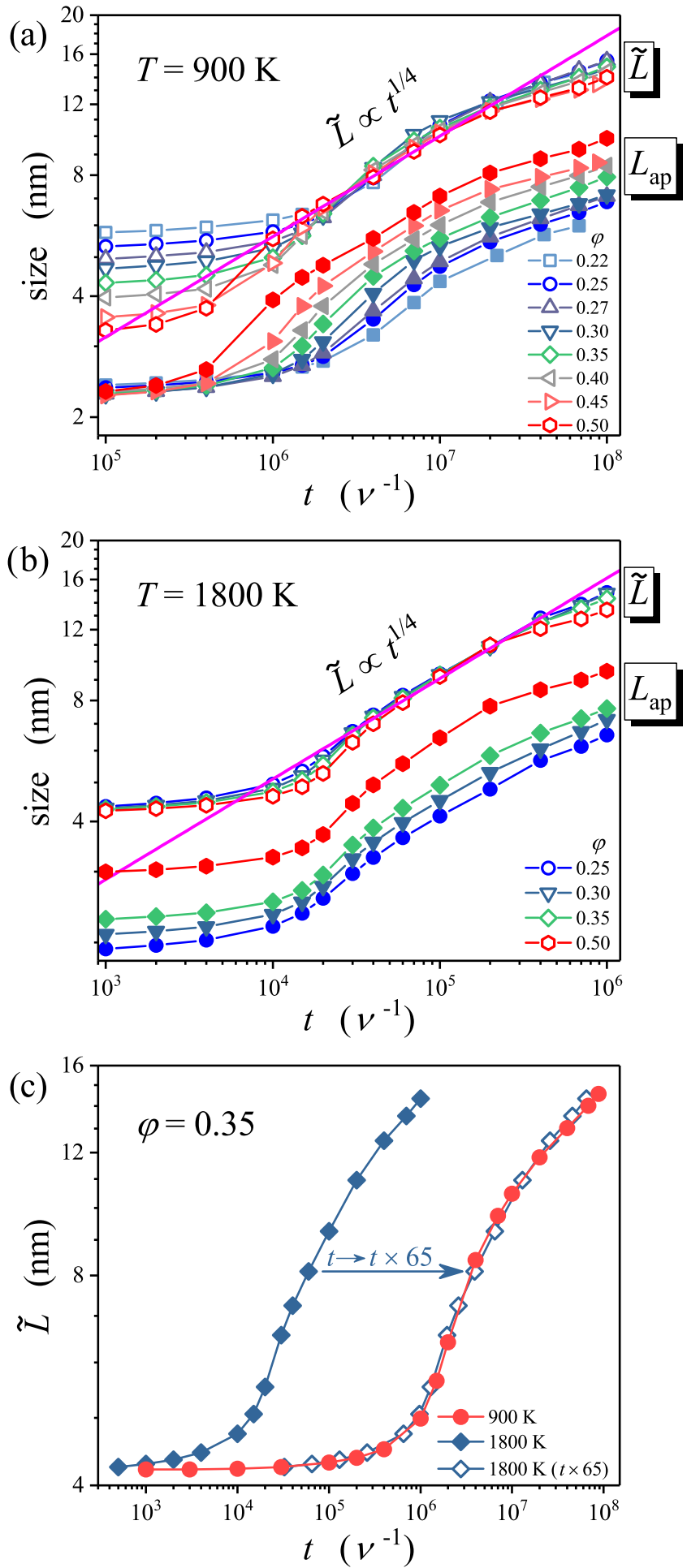

FIG. 3. Evolution of characteristic structure sizes with time, $t$. Networks with different solid fraction $\varphi$ at temperatures $T=900 \mathrm{~K}$ (a) and $1800 \mathrm{~K}$ (b). Closed symbols: apparent ligament diameter, $L_{\text {ap }}$; open symbols: characteristic spacing, $\tilde{L}$, between neighboring ligaments. Legends link symbol shapes to $\varphi$. Straight lines indicate $t^{1 / 4}$ kinetics (with arbitrary prefactor). Note universal behavior of $\tilde{L}$ and not of $L_{\text {ap }}$. (c) Comparison of $\tilde{L}$ versus $t$ at both temperatures for the structure with $\varphi=0.35$. Closed symbols: simulation results; open symbols: $1800 \mathrm{~K}$ results with the time axis multiplied by 65 . Note that this shift leads to superposition of the graphs. All axes in this figure have logarithmic scale. short times, $t$, the size does not yet increase. However (as discussed above), the surface morphology evolves from rough to more faceted. In the second stage, the coarsening law approximates the $t^{1 / 4}$ variation of size which would be expected for steady-state growth based on Ref. [53]. In the third stage, the coarsening slows down. We tentatively attribute that slowdown to an effect of the finite size of the simulation box-the microstructure evolution is no longer representative of bulk behavior once the ligament size approaches the box size.

The most striking observation in Fig. 3 is the different convergence behavior of the two size parameters. The evolution of $L_{\mathrm{ap}}$ depends on $\varphi$, even when all starting configurations have the same $L_{\mathrm{ap}}$. By contrast, the $\tilde{L}$ of all samples converge to a common graph, independent of $\varphi$, even when the initial $\tilde{L}$ differ. This implies that $\tilde{L}$-and not $L_{\text {ap }}$-is inherently the appropriate size parameter when discussing coarsening.

The coarsening at $1800 \mathrm{~K}$ is almost 2 orders of magnitude faster than at $900 \mathrm{~K}$. Besides the acceleration, and even though the surface morphologies-faceted versus roughdiffer between $900 \mathrm{~K}$ and $1800 \mathrm{~K}$, comparison of Figs. 3(a) and 3(b) shows that the qualitative features of the coarsening agree, independent of the temperature. For a closer inspection we focus on the simulations for $\varphi=0.35$, which have the identical initial structure at both temperatures. Figure 3(c) shows that the $1800 \mathrm{~K}$ graph of $\tilde{L}(t)$ here coincides quite precisely with the graph at $900 \mathrm{~K}$ if the time axis of the former is multiplied by 65 . The precise agreement implies the identical temperature dependence throughout the entire microstructure evolution. This is consistent with a single rate-limiting step and a unique value of the activation energy, namely $0.65 \mathrm{eV}$ (which is equivalent to 2.2 broken bonds).

With an eye on the possible power-law coarsening we performed one (very CPU-intensive) simulation run in which the simulation box had twice the standard edge length, namely 400 lattice spacings or $163.2 \mathrm{~nm}$, and 256 million sites. Temperature, solid fraction, and initial ligament spacing were $1800 \mathrm{~K}, 0.35$, and $4.7 \mathrm{~nm}$, respectively. Figure 4(a) shows a log-log plot of the results for $\tilde{L}(t)$ versus $t$, superimposed to those obtained with the standard size box (edge length 200 lattice spacings) under otherwise identical conditions. It is obvious that the slowdown in the third stage of coarsening in Fig. 3 is only seen for the smaller box; this feature is indeed an artifact of the box size. The simulations with the larger box also confirm the agreement of the simulation with the $t^{1 / 4}$ coarsening law. This agreement is further supported by the linearity of the graph of $\tilde{L}^{4}$ versus $t$ in Fig. 4(b). Analogous graphs for the remaining simulation runs (see the Supplemental Material [52]) also show essentially linear behavior in the second stage of the coarsening.

\section{Evolution of network connectivity}

Next we inspect the evolution of the network topology during coarsening. Figures 5(a) and 5(b) show the genus $G$ of the largest cluster and the number, $B_{0}$, of connected components, respectively, of each stage during coarsening at 

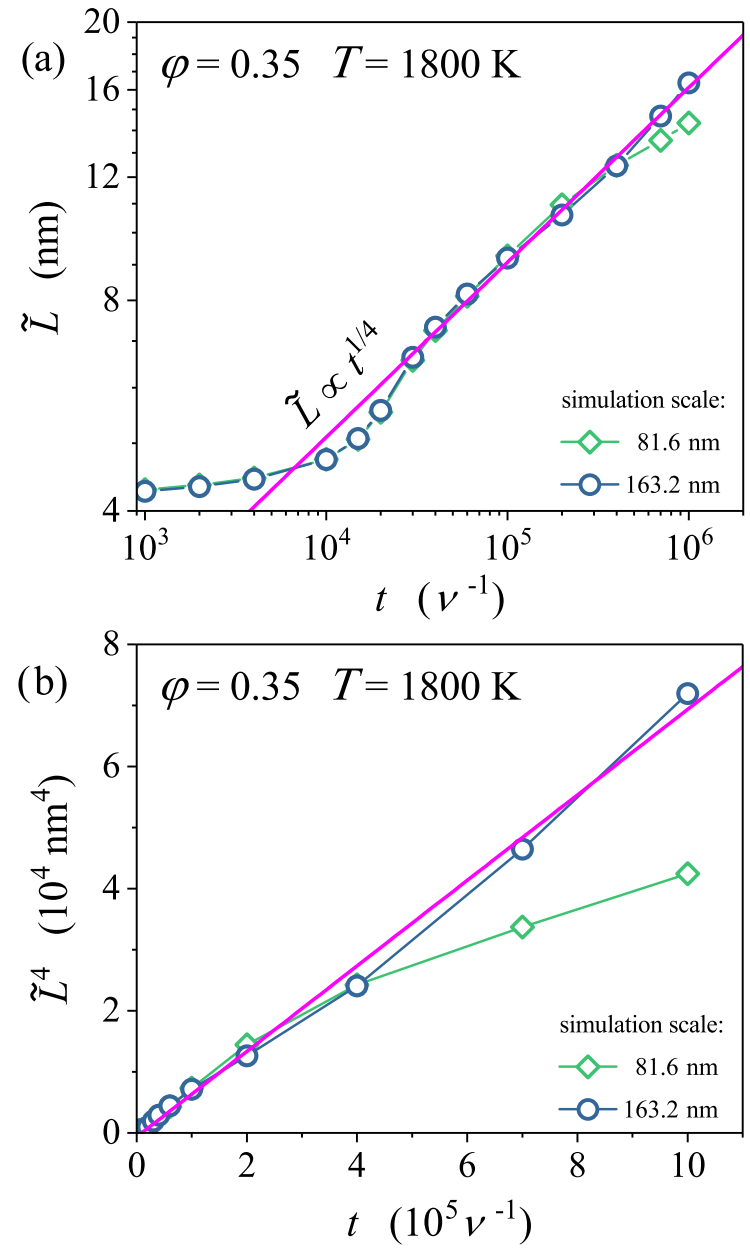

FIG. 4. Coarsening kinetics. For the example of $T=1800 \mathrm{~K}$ and $\varphi=0.35$, panel (a) shows log-log plot of characteristic ligament spacing $\tilde{L}$ versus time $t$. Open symbol, standard size simulation box with edge length $81.6 \mathrm{~nm}$; closed symbols, larger simulation box with edge length $163.2 \mathrm{~nm}$. Solid line: $t^{1 / 4}$ kinetics (with arbitrary prefactor). Slowdown of coarsening at largest $\tilde{L}$ for the small box is an artifact of the finite box size. (b) Same data, plotted as $\tilde{L}^{4}$ versus $t$. Note the linear variation for the results obtained with the larger box size. Data in this figure confirm that the coarsening law of the simulation is inherently $\tilde{L} \propto t^{1 / 4}$.

$900 \mathrm{~K}$. The results at $1800 \mathrm{~K}$ (not shown) are consistent, except that few (a fraction $\sim 10^{-5}$ of all sites) free atoms in the gas here contribute to $B_{0}$. In Fig. 5(a), the initial values of $G$ depend on $\varphi$. This is a consequence of, first, the different $H$, and, second, the variation of connectivity of leveled-wave structures with $\varphi$, see below. Most importantly, it is seen that the values of $G$ systematically drop when the coarsening sets in. That drop is faster at lesser $\varphi$. The final $G$ value of the structure with $\varphi=0.22$ approaches zero, indicating that this sample has almost completely disintegrated into isolated dense clusters. One of the pinch-off events which decrease $G$ is visualized in the inset in Fig. 5(a).

The appearance of isolated clusters may also be followed by inspection of the number of connected components, Fig. 5(b). For samples with $\varphi>0.35$ that number changes little or retains its initial value of 1 . By contrast, $B_{0}$ of samples with lesser $\varphi$ increases significantly during coarsening.
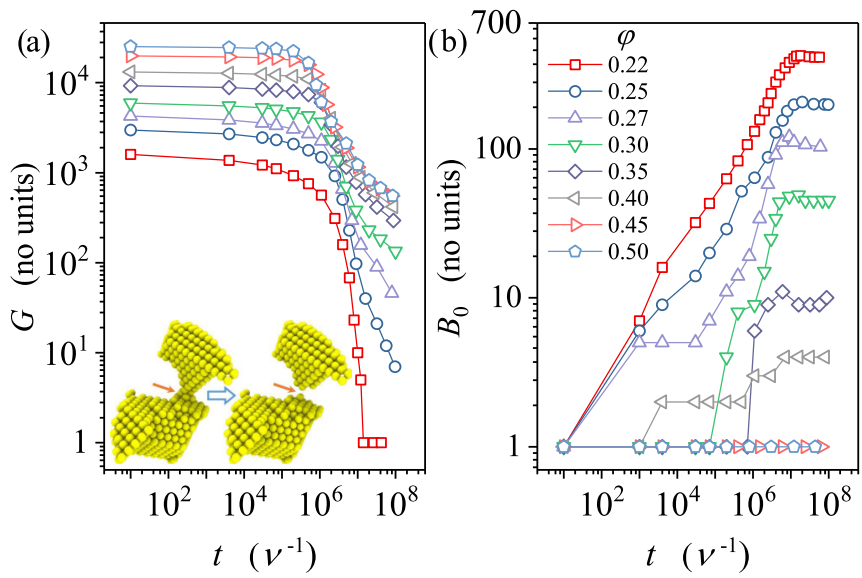

FIG. 5. Evolution of the topology during coarsening, here for the $900 \mathrm{~K}$ simulation. (a) Topological genus, $G$, of the largest cluster versus time, $t$, for structures of different solid fraction $\varphi$. Inset exemplifies a pinch-off event. (b) Number, $B_{0}$, of connected components versus $t$. Note logarithmic axis. Legend in (b) links symbol shapes to solid fraction, $\varphi$.

For the $900 \mathrm{~K}$ simulation, Fig. 6 inspects how the scaled genus $g$ [Eq. (5)] of the largest cluster in each state varies with the solid fraction $\varphi$. The bold line refers to the leveled-wave geometry that underlies our initial structures, where

$$
g=\frac{2 \pi \alpha^{3}}{3 \sqrt{3}}\left(1-\xi^{2}\right) e^{-\xi^{2} / 2}
$$

with $\xi=\sqrt{2} \operatorname{erf}^{-1}(2 \varphi-1)$ [21]. The symbols refer to the numerical results obtained by analysis of the discrete atomic structures. In the initial state $\left(\tilde{L}=\tilde{L}_{0}\right)$, the numerical analysis underestimates $g$. This can be understood as the result of $\tilde{L}$

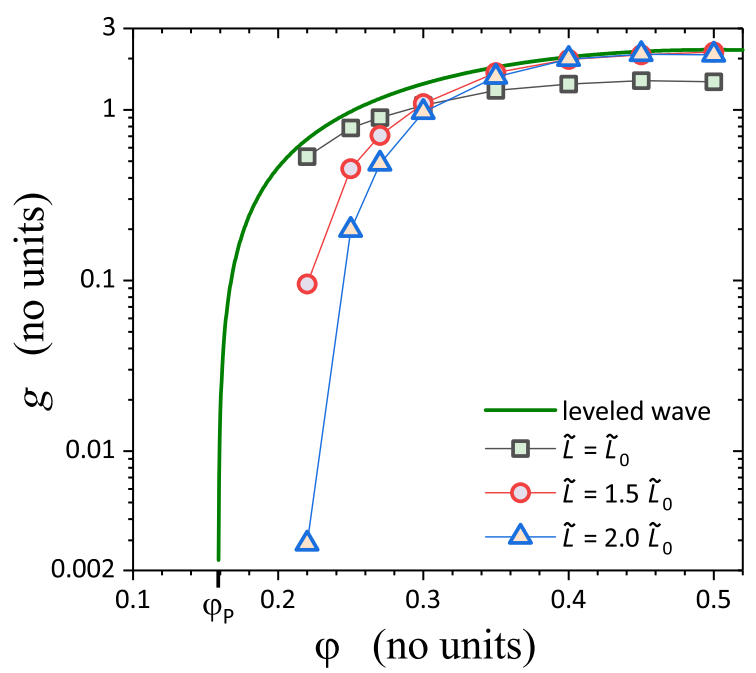

FIG. 6. Scaled genus, $g$, versus solid fraction $\varphi$ in different stages of the coarsening. Bold solid line: Theory for leveled wave initial structure, Eq. (6); $\varphi_{P}$ denotes the percolation threshold of the theory. Symbols: Numerical results for initial structures $\left(\tilde{L}=\tilde{L}_{0}\right)$ and after coarsening to $\tilde{L}=1.5 \tilde{L}_{0}$ and to $\tilde{L}=2.0 \tilde{L}_{0}$, see legend. Note that $g$ of structures with $\varphi>0.3$ converge toward the theory, whereas structures with $\varphi<0.3$ move away from it. 


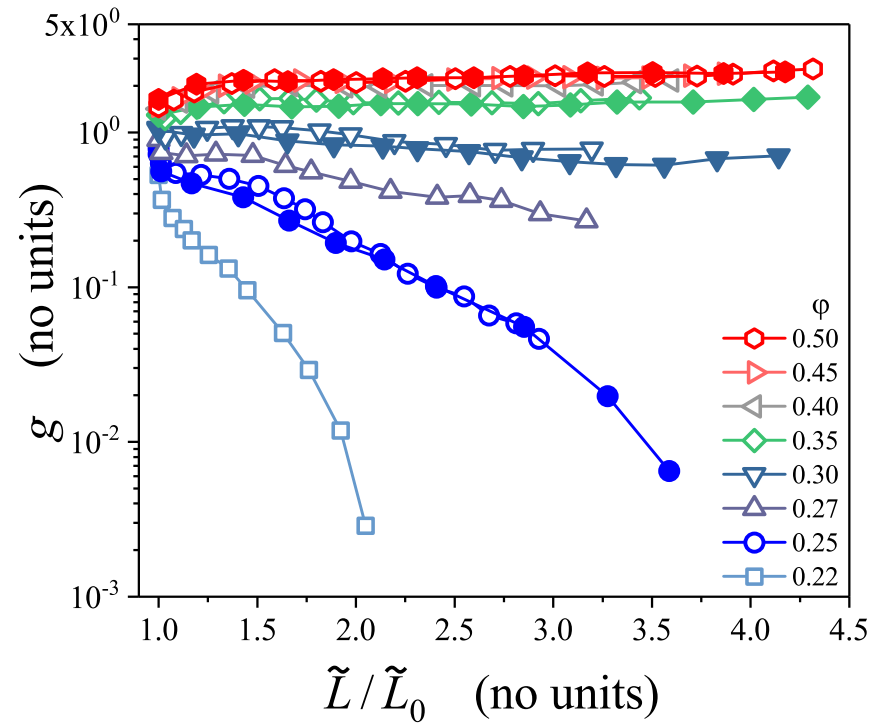

FIG. 7. Scaled genus, $g$, versus characteristic spacing, $\tilde{L}$, between neighboring ligaments. $\tilde{L}$ has been normalized to initial value, $\tilde{L}_{0}$, before the onset of coarsening. Different symbol shapes discriminate between different values of solid fraction, $\varphi$, as indicated in legend. Open symbols: coarsening at $900 \mathrm{~K}$; closed symbols: $1800 \mathrm{~K}$. Note that structures with $\varphi<0.3$ tend to lose connectivity on coarsening.

being underestimated by the surface reconstruction algorithm, as discussed above. As the ligaments grow to $\tilde{L}=1.5 \tilde{L}_{0}$ and then to $\tilde{L}=2.0 \tilde{L}_{0}$, the scaled genus of structures with $\varphi>0.3$ relaxes toward the theory line, which it accurately meets. In other words, the topology of the leveled wave appears stable during coarsening at those solid fractions. By contrast, structures with $\varphi<0.3$ evolve away from the leveled wave topology during coarsening. The more $\varphi$ approaches the percolation threshold of the theory $\left(\varphi_{P}=\frac{1}{2}\left[1-\operatorname{erf}\left(\sqrt{2}^{-1}\right)\right] \approx\right.$ 0.16 [21]), the faster connectivity is lost during coarsening.

These observations are further illustrated by the graphs, in Fig. 7, of $g$ versus $\tilde{L}$ for the structures with various $\varphi$ and at both temperatures. All $\tilde{L}$ in this figure are scaled to the respective initial value, $\tilde{L}_{0}$. It is seen that the data from the $900 \mathrm{~K}$ runs (open symbols) and from the $1800 \mathrm{~K}$ runs (closed symbols) are in excellent agreement. Furthermore, the figure confirms that $g$ of structures with $\varphi \geqslant 0.3$ varies little during coarsening. This is at least consistent with self-similar coarsening at those solid fractions. Remarkably, however, samples with $\varphi<0.3$ exhibit a decrease of $g$ on coarsening. That trend is weak at $\varphi=0.27$ and it becomes more prominent as $\varphi$ decreases further. For example, the value $g$ of the sample with $\varphi=0.25$ decreases by the factor 110 between the initial and final data point. These findings are consistent at the two temperatures of our study. In other words, within the range of our simulations, there appears to be a transition between apparently self-similar coarsening for $\varphi \geqslant 0.3$ and a behavior with progressive loss of connectivity and eventually a percolation-to-cluster transition for $\varphi<0.3$. The renderings of the structural evolution in real space in the Supplemental Material [52] visualize the geometry changes behind this loss of connectivity.

\section{DISCUSSION}

\section{A. Assessing the initial microstructures}

Our study is motivated by experimental observations on coarsening of nanoporous gold made by dealloying. The two processes, dealloying and coarsening, are distinctly different: During dealloying, chemical or electrochemical driving forces for the dissolution of the less-noble element let the porosity and the area of surface increase over time. Coarsening, by contrast, is driven by the strife to reduce the net excess surface energy that was introduced during dealloying. During this process, porosity is conserved and the area of surface decreases over time. As their starting point, our simulations of coarsening require a realistic model for the microstructures that are produced by dealloying. The leveled random fields which provide these initial structures in our study approximate the microstructure at the end of early-stage spinodal decomposition [36]. Thereby, our results are also relevant for the microstructure evolution during the coarsening of spinodal structures, in other words, for late-stage spinodal decomposition.

Because dealloying and early-stage spinodal decomposition involve distinctly different processes, it is not a priori obvious that our initial structures provide useful approximations to the microstructure of NPG. Yet Ref. [21] points out the excellent agreement of the elastic properties and of the scaled genus of leveled random fields with experiments on NPG. To date, no other microstructural model has received a comparable validation by comparison to experiment. This motivates and supports the initial configurations of our study.

\section{B. Microscopic details of the KMC simulation}

Our KMC approach is based on a simplified representation of the experimental scenario. Its rigid lattice forbids the atomic-scale relaxation of the surface atoms as well as the long-range elastic and plastic deformation of the bulk in response to capillary forces. The activation energies of the individual diffusion events are embodied in a simplified manner and the possible impact of adsorbates ignored. Instead, the model focusses on the most essential features behind the coarsening of metal network structures, specifically the (anisotropic) excess energy of the surface and the transport by diffusion along the surface and along step edges and not through the bulk. In support of the adequateness of this model, the KMC simulations in Refs. [31,32,54] have obtained semiquantitative agreement with experiments on various aspects of the structural evolution during the making of NPG by dealloying.

Previous KMC studies of NPG have used bond energy values of $-0.285 \mathrm{eV}$ [31] and $-0.15 \mathrm{eV}[32,54]$. The present value of $-0.30 \mathrm{eV}$ is motivated by the ensuing, realistic values of the surface tensions, see Sec. II. Furthermore, the value, $0.65 \mathrm{eV}$, of the apparent activation energy for coarsening in the present work compares favorably to $0.64 \mathrm{eV}$ for gold surface diffusion in vacuum [55], a value that was also found to agree well with the activation energy for nanoporous gold coarsening in air near $900 \mathrm{~K}$ [24]. The reasonable value of the activation energy supports our choice of the bond energy value. 
In view of its simplified representation of diffusion, our KMC simulation is not designed for a quantitative representation of coarsening timescales. Yet one may ask whether the simulation reproduces the coarsening rate in a roughly realistic manner. If we adopt the value $v=10^{12} \mathrm{~s}^{-1}$ (see Sec. II B) for the attempt frequency, then the time interval of $10^{8} v$ for coarsening from 4 to $16 \mathrm{~nm}$ at $900 \mathrm{~K}$ [see Fig. 3(c)] corresponds to $100 \mu \mathrm{s}$. Extrapolation using the activation energy of $0.65 \mathrm{eV}$ suggests that the same process would take slightly more than half an hour at room temperature. In view of the fact that coarsening behind the dealloying front (secondary dealloying $[54,56]$ ) in NPG leads to ligament sizes of 20-40 nm after several hours of dealloying at room temperature [34], the time constant appears reasonable.

\section{Kinetics of coarsening and appropriate size parameter}

Our findings for the microstructure evolution by surface transport emphasize that the characteristic spacing between ligament centers, $\tilde{L}$, is more inherently linked to the coarsening kinetics than the ligament diameter, $L_{\mathrm{ap}}$. This is of relevance since many experiments on NPG parametrize the characteristic microstructural length scale by a mean ligament diameter $L$ - similarly to our $L_{\mathrm{ap}}$-which is determined by measuring dimensions in electron micrographs. Other experiments compute $L$ as the inverse of the specific surface area, which is accessible through electrochemistry. The more fundamental parameter $\tilde{L}$ relates to an underlying wavelength and can therefore be determined from information in reciprocal space. Experiment provides such information in the form of peak positions in small-angle scattering $[37,38,57]$ or in Fourier transforms of electron micrographs [23]. Yet $\tilde{L}$ is rarely reported in experimental studies.

We find the coarsening kinetics consistent with the $t^{1 / 4}$ power law that is expected [53] for coarsening mediated by surface transport. The experimental literature for NPG provides no conclusive picture on the time exponent. The reported data are typically not inconsistent with exponent $1 / 4$, but confidence limits are wide and some data appear even consistent with linear growth $[23,24,58]$. Conceivably, more precise data may be obtained by basing future analysis systematically on $\tilde{L}$.

\section{Impact of faceting}

Slender cylindrical rods with surface energy are susceptible to pinch-off by the Plateau-Rayleigh instability as, for ratios of length over diameter $>\pi$, small fluctuations in diameter reduce the energy and can spontaneously grow [59]. In networks, as in the present study, matter can be redistributed over distances larger than the length (distance between nodes connecting neighboring ligaments) of ligaments and so the instability is expected even when, at high $\varphi$, the ligaments appear short and thick. Indeed, pinch-off events are observed in our structures at all solid fractions.

The Plateau-Rayleigh instability is affected by anisotropy of the specific surface free energy, $\gamma$, and may slow down when there are steep cusps in $\gamma$ of crystal surfaces at lowindex orientations [60]. The cusps prevent the rotation of terraces out of their low index/low energy orientation, thereby suppressing the fluctuations that initiate the instability. The presence of facets, which are a consequence of steep cusps in $\gamma$, may also affect coarsening exponents [61]. It is therefore noteworthy that our structures at $900 \mathrm{~K}$ appear almost perfectly faceted, indicating steep cusps in $\gamma$. The enhanced roughness at $1800 \mathrm{~K}$ can be understood as the consequence of the specific step edge excess free-energy (line tension) decreasing at higher temperature due to the configurational entropy that is associated with step-edge fluctuations. The decrease of the step-edge energy lets the cusps become more shallow. Significantly, we observe no qualitative changes in the coarsening behavior between the two temperatures, in spite of the substantially different degrees of faceting or roughness. We note the abundance of kink sites in all structures, even in the equilibrated small particles of Fig. 1. The number of atoms in those particles happens not to be suitable for forming a perfectly faceted outer surface, and some kinks are then configurationally necessary. In the network structures, the kinks provide for facile migration of step edges as an alternative pathway for microstructure evolution. This pathway differs from the coordinated rotation of the terraces out of their low index orientation, as it is considered in Ref. [60].

High-resolution transmission electron micrographs of the surface of NPG have revealed microfacets [62], and faceting on the scale of the ligament size has been reported in several studies of coarsened NPG [24,63,64]. This appears natural, since the Wulff shape of gold is known to retain facets up to temperatures close to the melting point [65]. However, many other studies of NPG present scanning electron micrographs that show curved and, hence, rough surfaces [13-15,17,58]. Thus, the faceting in our simulation is not unanimously supported by experiment. Yet the observations on the time exponent for coarsening are consistent in simulations and experiment. In this respect it is significant that our simulation finds the coarsening kinetics as independent of the degree of faceting. Even though the confidence limits of experiment and of simulation are wide, our simulation apparently catches essential features of experiments on NPG, irrespective of the faceting.

The texture in the orientation of the ligaments of our coarsened samples represents another aspect which is not typically reported for experimental coarsened NPG. Yet strikingly similar textures have been reported in one experimental study of NPG [66] and in a study of dealloyed nanoporous silver [67].

\section{E. Impact of solid fraction}

The loss of connectivity during coarsening of spinodal structures is of particular relevance in polymer blends, where extended coarsening can entail a percolation-to-cluster transition [44,68-71]. The transition can be limited to strongly asymmetric $(\varphi \ll 0.5)$ blends [71] but can also occur at any phase fraction [69]. Studies of the topology evolution during spinodal coarsening in experiment $[44,72]$ as well as simulation [49] have documented the loss of connections. The scaled connectivity has been reported in an experimental study of polymer blends [44], which finds $g$ invariant during coarsening at $\varphi=0.5$, whereas a 4 -fold increase in domain size brings a 10 -fold reduction in $g$ when $\varphi=0.35$. This 
observation supports the trends in our simulation. In the phase-field study of Ref. [29], spinodally decomposed (by bulk diffusion) mixtures with phase fractions $\varphi=0.5,0.40$, and 0.36 are bicontinuous and coarsen self-similarly, whereas mixtures with $\varphi=0.30$ and 0.22 are not percolating at any stage of the decomposition process.

As a central observation of the present study, the evolution of scaled genus during coarsening by surface diffusion depends strongly on the solid fraction. For $\varphi \geqslant 0.3, g$ remains sensibly invariant during coarsening. At lower solid fraction, by contrast, $g$ decreases when ligaments coarsen. The structure with the smallest $\varphi$ even disintegrates completely when the characteristic structure size reaches twice the initial value. Our observations on surface-transport-mediated coarsening of network structures resembling dealloyed nanoporous metals thereby have parallels to the findings for spinodal decomposition by bulk diffusion in Ref. [29]. Yet, at $\varphi<0.36$, the present network structures exhibit substantially enhanced stability compared to the spinodal ones. It is therefore significant that our findings are well compatible with the experimental observations of Ref. [44] on the evolution of the scaled genus in spinodally decomposed polymer blends.

As our study aims to explore the connectivity evolution during coarsening of NPG, we now summarize the relevant experimental findings on that material. We focus on the initial stages (to $L \sim 100 \mathrm{~nm}$ ) of coarsening. Data for the variation of the effective Young's modulus during coarsening of NPG with $\varphi=0.44,0.35$, and 0.27 have been discussed, respectively, in terms of conservation and slow loss and rapid loss of connectivity $[14,18]$. The more direct analysis of tomographic reconstructions has been discussed in terms of conserved connectivity at $\varphi \approx 0.30[15,27]$. Yet, even though error bars are significant, an initial, slow loss in connectivity - as may also be perceived at $\varphi=0.30$ in our Fig. 7-is not inconsistent with that latter data. ${ }^{1}$ It is seen that the experimental trends agree qualitatively with those of our simulation. In particular, our results provide a direct confirmation of the trends inferred more indirectly from the elasticity data of Refs. $[14,18]$. Furthermore, while the essentially constant connectivity in our results for $\varphi=0.30$ is consistent with the suggestion, in Refs. [15,27], of self-similar coarsening at $\varphi$ near 0.30 , our results also suggest that self-similarity will be strongly lost when the solid fraction is decreased even slightly below that value.

The link between $\varphi$ and the variation (or not) of $g$ during coarsening may be qualitatively understood as the consequence of a competition between the rate of ligaments breaking and the rate at which matter is transported from the broken ligaments onto the surviving ones [14]. Thinner ligaments at lower $\varphi$ are expected to undergo faster pinch-off. Yet, for a given value of $\tilde{L}$, the diffusion distance to the neighboring, surviving ligaments is independent of $\varphi$. The time for redistributing the material from the broken ligaments to the neighbors may therefore not sensitively depend on $\varphi$. Thus, at any given instant, structures with lesser solid fraction

\footnotetext{
${ }^{1}$ At any $\varphi$, experiment indicates a slow increase of connectivity on further coarsening, beyond $L=100 \mathrm{~nm}[14,15,18]$. This has been linked to densification by spontaneous plastic deformation [14].
}

may contain more dangling ligaments and so effectively have lesser connectivity.

\section{CONCLUSIONS}

Using leveled-wave microstructures of different solid fraction $\varphi$ as models for as-dealloyed nanoporous gold, we have followed the evolution of the structure size and of the topology during coarsening by surface diffusion. We find that the value of $\varphi$ is decisive for the evolution of the topology. Structures with $\varphi \geqslant 0.30$ maintain their connectivity during coarsening, while those with $\varphi<0.30$ become more and more disconnected as the coarsening proceeds. This process is slow for $\varphi$ only little below 0.30 and it accelerates as $\varphi$ approximates the percolation threshold, $\varphi=0.16$, of the leveled-wave model. Earlier work on coarsening of spinodally decomposed structures using phase-field simulation found disconnected structures at all solid fractions below 0.36. By contrast, our results agree with experiment in showing sensibly constant $g$ down to $\varphi=0.30$. They are also consistent with experiments which find that connected structures with much lesser $\varphi$ than 0.36 emerge from dealloying and remain load-bearingthough with a gradual degradation of the mechanical behavior that implies diminishing connectivity-during extended coarsening.

Our results confirm suggestions $[1,13,14]$ that the strong dependency of the apparent strength of the ligaments in NPG on the ligament size in some experimental studies may be artifacts. When the ligament size is tuned by coarsening, the connectivity will decrease and this needs to be accounted for when computing the local strength of ligaments based on empirical data for the effective macroscopic strength of the network. We note that this observation is not restricted to experimental data. The connectivity of bicontinuous structures generated by spinodal decomposition may be substantially less than that of the leveled-wave model, which compares favorably to experimental NPG. Numerical studies using spinodally decomposed structures as their microstructure model may therefore also work with unrealistically small connectivity. The data for strength and stiffness of such studies may therefore require verification.

Our study has implications for designing nanoscale metal network structures as lightweight structural materials and for the discussion of the behavior of NPG in the context of smallscale plasticity. Good mechanical properties appear to require structures with solid fraction at or above 0.3; lesser solid fractions invite loss of connectivity or even disintegration. This is of relevance because many experimental studies have so far worked with $\varphi$ between 0.25 and 0.30 . A purposeful selection of the solid fraction provides opportunities for improved mechanical behavior in dealloying-made nanoporous metal.

\section{ACKNOWLEDGMENT}

This work was supported by Deutsche Forschungsgemeinschaft, Projektnummer 192346071-SFB 986. 
[1] H.-J. Jin, J. Weissmüller, and D. Farkas, MRS Bull. 43, 35 (2018).

[2] J. Biener, A. M. Hodge, A. V. Hamza, L. M. Hsiung, and J. H. Satcher, Jr., J. Appl. Phys. 97, 024301 (2005).

[3] C. Volkert, E. Lilleodden, D. Kramer, and J. Weissmüller, Appl. Phys. Lett. 89, 061920 (2006).

[4] J. Biener, A. M. Hodge, J. R. Hayes, C. A. Volkert, L. A. Zepeda-Ruiz, A. V. Hamza, and F. F. Abraham, Nano Lett. 6, 2379 (2006).

[5] A. Hodge, J. Biener, J. Hayes, P. Bythrow, C. Volkert, and A. Hamza, Acta Mater. 55, 1343 (2007).

[6] H.-J. Jin, L. Kurmanaeva, J. Schmauch, H. Rösner, Y. Ivanisenko, and J. Weissmüller, Acta Mater. 57, 2665 (2009).

[7] N. J. Briot, T. Kennerknecht, C. Eberl, and T. J. Balk, Phil. Mag. 94, 847 (2014).

[8] H. J. Jin and J. Weissmüller, Science 332, 1179 (2011).

[9] N. Mameka, J. Markmann, and J. Weissmüller, Nat. Commun. 8, 1976 (2017).

[10] L. Lührs, B. Zandersons, N. Huber, and J. Weissmüller, Nano Lett. 17, 6258 (2017).

[11] S. Sun, X. Chen, N. Badwe, and K. Sieradzki, Nat. Mater. 14, 894 (2015)

[12] N. Badwe, X. Chen, D. Schreiber, M. Olszta, N. Overman, E. Karasz, A. Tse, S. Bruemmer, and K. Sieradzki, Nat. Mater. 17, 887 (2018).

[13] N. Mameka, K. Wang, J. Markmann, E. T. Lilleodden, and J. Weissmüller, Mater. Res. Lett. 4, 27 (2016).

[14] L.-Z. Liu, X.-L. Ye, and H.-J. Jin, Acta Mater. 118, 77 (2016).

[15] K. Hu, M. Ziehmer, K. Wang, and E. T. Lilleodden, Philos. Mag. 96, 3322 (2016).

[16] J. Erlebacher, Phys. Rev. Lett. 106, 225504 (2011).

[17] K. Mangipudi, E. Epler, and C. Volkert, Acta Mater. 140, 337 (2017).

[18] L.-Z. Liu and H.-J. Jin, Appl. Phys. Lett. 110, 211902 (2017).

[19] N. Huber, Front. Mater. 5, 69 (2018).

[20] B.-N. D. Ngô, A. Stukowski, N. Mameka, J. Markmann, K. Albe, and J. Weissmüller, Acta Mater. 93, 144 (2015).

[21] C. Soyarslan, S. Bargmann, M. Pradas, and J. Weissmüller, Acta Mater. 149, 326 (2018).

[22] R. Li and K. Sieradzki, Phys. Rev. Lett. 68, 1168 (1992).

[23] L. Qian and M. Chen, Appl. Phys. Lett. 91, 083105 (2007).

[24] Y.-c. K. Chen-Wiegart, S. Wang, Y. S. Chu, W. Liu, I. McNulty, P. W. Voorhees, and D. C. Dunand, Acta Mater. 60, 4972 (2012).

[25] D. Farkas, A. Caro, E. Bringa, and D. Crowson, Acta Mater. 61, 3249 (2013).

[26] X.-Y. Sun, G.-K. Xu, X. Li, X.-Q. Feng, and H. Gao, J. Appl. Phys. 113, 023505 (2013).

[27] H. Jeon, N.-R. Kang, E.-J. Gwak, J.-i. Jang, H. N. Han, J. Y. Hwang, S. Lee, and J.-Y. Kim, Scr. Mater. 137, 46 (2017).

[28] Y. Kwon, K. Thornton, and P. W. Voorhees, Phys. Rev. E 75, 021120 (2007).

[29] Y. Kwon, K. Thornton, and P. W. Voorhees, Philos. Mag. 90, 317 (2010).

[30] L. Banas, A. Novick-Cohen, and R. Nuernberg, Netw. Heterogen. Media 8, 37 (2013).

[31] J. Erlebacher, M. J. Aziz, A. Karma, N. Dimitrov, and K. Sieradzki, Nature 410, 450 (2001).

[32] J. Erlebacher, J. Electrochem. Soc. 151, C614 (2004).

[33] V. E. Brunini, C. A. Schuh, and W. C. Carter, Phys. Rev. E 83, 021119 (2011).
[34] M. Graf, B. Roschning, and J. Weissmüller, J. Electrochem. Soc. 164, C194 (2017).

[35] S. Parida, D. Kramer, C. A. Volkert, H. Rösner, J. Erlebacher, and J. Weissmüller, Phys. Rev. Lett. 97, 035504 (2006).

[36] J. W. Cahn, J. Chem. Phys. 42, 93 (1965).

[37] C. J. Dotzler, B. Ingham, B. N. Illy, K. Wallwork, M. P. Ryan, and M. F. Toney, Adv. Funct. Mater. 21, 3938 (2011).

[38] B. Lin, L. Kong, P. D. Hodgson, S. Mudie, A. Hawley, and L. F. Dumée, RSC Adv. 7, 10821 (2017).

[39] C. C. Battaile, Comput. Methods Appl. Mech. Eng. 197, 3386 (2008).

[40] S. Plimpton, C. Battaile, M. Chandross, L. Holm, A. Thompson, V. Tikare, G. Wagner, E. Webb, X. Zhou, C. G. Cardona et al., Sandia Report SAND 2009-6226 (2009), https://prod-ng.sandia.gov/techlib-noauth/access-control.cgi/ 2009/096226.pdf.

[41] A. Stukowski, Modell. Simul. Mater. Sci. Eng. 18, 015012 (2009).

[42] J. Koettgen, T. Zacherle, S. Grieshammer, and M. Martin, Phys. Chem. Chem. Phys. 19, 9957 (2017).

[43] Y. Umeno, C. Elsässer, B. Meyer, P. Gumbsch, M. Nothacker, J. Weissmüller, and F. Evers, Eurphys. Lett. 78, 13001 (2007).

[44] C. R. Lopez-Barron and C. W. Macosko, Langmuir 25, 9392 (2009).

[45] S. Cattarin, D. Kramer, A. Lui, and M. M. Musiani, J. Phys. Chem. C 111, 12643 (2007).

[46] C. Lakshmanan, R. N. Viswanath, S. R. Polaki, R. Rajaraman, S. Dash, and A. K. Tyagi, Electrochim. Acta 182, 565 (2015).

[47] A. Stukowski, JOM 66, 399 (2014).

[48] E. T. Lilleodden and P. W. Voorhees, MRS Bull. 43, 20 (2018).

[49] M. Gameiro, K. Mischaikow, and T. Wanner, Acta Mater. 53, 693 (2005).

[50] T. Kaczynski, K. Mischaikow, and M. Mrozek, Computational Homology (Springer, Berlin, 2006).

[51] K. Mischaikow, H. Kokubu, M. Mrozek, P. Pilarczyk, T. Gedeon, J.-P. Lessard, and M. Gameiro, software available at http://chomp.rutgers.edu (2014).

[52] See Supplemental Material at http://link.aps.org/supplemental/ 10.1103/PhysRevMaterials.3.076001 for parameters used in generating the initial microstructures, for additional graphs on size evolution with time, and for visualization of initial as well as coarsened microstructures.

[53] C. Herring, J. Appl. Phys. 21, 301 (1950).

[54] T. Krekeler, A. V. Straßer, M. Graf, K. Wang, C. Hartig, M. Ritter, and J. Weissmüller, Mater. Res. Lett. 5, 314 (2017).

[55] I. Beszeda, I. Szabo, and E. Gontier-Moya, Appl. Phys. A 78, 1079 (2004).

[56] X.-L. Ye, N. Lu, X.-J. Li, K. Du, J. Tan, and H.-J. Jin, J. Electrochem. Soc. 161, C517 (2014).

[57] M. Graf, B.-N. D. Ngô, J. Weissmüller, and J. Markmann, Phys. Rev. Mater. 1, 076003 (2017).

[58] S. Kuwano-Nakatani, T. Fujita, K. Uchisawa, D. Umetsu, Y. Kase, Y. Kowata, K. Chiba, T. Tokunaga, S. Arai, Y. Yamamoto et al., Mater. Trans. 56, 468 (2015).

[59] Lord Rayleigh, Proc. Lond. Math. Soc. s1-10, 4 (1878).

[60] J. W. Cahn, Scr. Metall. 13, 1069 (1979).

[61] S. Biagi, C. Misbah, and P. Politi, Phys. Rev. E 89, 062114 (2014). 
[62] T. Fujita, P. Guan, K. McKenna, X. Lang, A. Hirata, L. Zhang, T. Tokunaga, S. Arai, Y. Yamamoto, N. Tanaka, Y. Ishikawa, N. Asao, Y. Yamamoto, J. Erlebacher, and M. Chen, Nat. Mater. 11, 775 (2012).

[63] F. Kertis, J. Snyder, L. Govada, S. Khurshid, N. Chayen, and J. Erlebacher, JOM 62, 50 (2010).

[64] Y. Sun, S. A. Burger, and T. J. Balk, Philos. Mag. 94, 1001 (2014).

[65] H. Sadan and W. D. Kaplan, J. Mater. Sci. 41, 5099 (2006).

[66] I. McCue, E. Benn, B. Gaskey, and J. Erlebacher, Annu. Rev. Mater. Res. 46, 263 (2016).
[67] C. Wang and Q. Chen, Chem. Mater. 30, 3894 (2018).

[68] J. Läuger, R. Lay, and W. Gronski, J. Chem. Phys. 101, 7181 (1994).

[69] J. Lee and C. Han, Polymer 40, 2521 (1999).

[70] I. Demyanchuk, S. A. Wieczorek, and R. Holyst, J. Chem. Phys. 121, 1141 (2004).

[71] D. Y. Hsu, C. M. Chou, C. Y. Chuang, and P. D. Hong, ACS Macro Lett. 4, 1341 (2015).

[72] J. M. Hyde, M. K. Miller, M. G. Hetherington, A. Cerezo, G. D. W. Smith, and C. M. Elliott, Acta Metall. Mater. 43, 3415 (1995). 